\title{
Emprendimiento en épocas de crisis: Un análisis exploratorio de los efectos de la COVID-19
}

\author{
Entrepreneurship in times of crisis: An exploratory analysis of the \\ COVID-19's effects
}

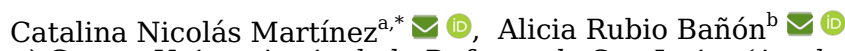

a) Centro Universitario de la Defensa de San Javier (Academia General del Aire) (España)

b) Universidad de Murcia (España)

* Contacto principal: catalina.nicolas@cud.upct.es (Catalina Nicolás Martínez)

\begin{abstract}
Resumen
La pandemia actual -COVID19- ha llevado a muchos gobiernos a tomar medidas drásticas en respuesta a la crisis de salud global. Las medidas de bloqueo como respuesta a la crisis han frenado aún más las actividades económicas a nivel mundial. En este contexto destaca la importancia del papel de los emprendedores para acelerar la recuperación económica. Este trabajo se centra en analizar cómo los emprendedores enfrentan la situación causada por la COVID-19 a través de un estudio cuantitativo con una muestra de 236 emprendedores españoles ubicados geográficamente en la Región de Murcia. Los resultados muestran cómo los emprendedores han tenido que adaptarse a estas nuevas circunstancias enfrentándose a desafíos como la necesidad de digitalización, la falta de financiamiento, la disminución del capital humano o la percepción de nuevas oportunidades que se conviertan en negocios viables.

Palabras clave: emprendimiento; COVID-19; crisis económica; desafíos; políticas de estímulo del emprendimiento Clasificación JEL: I18; L21; L23; L26

Abstract

The current pandemic (COVID19) have led many governments to take drastic measures in respond of the global health crisis. The lockdown measures as a response to the crisis has further slowed global economic activities. In this context highlights the importance of the role of entrepreneurs to accelerate economic recovery. This paper focuses on analyzing how entrepreneurs face the situation caused by COVID-19 through a quantitative study with a sample of 236 Spanish entrepreneurs from the Region of Murcia. Results show how entrepreneurs have had to adapt to these new circumstances, facing challenges regarding the need for digitization, the lack of financing, the decrease in human capital or the perception of new opportunities that become viable businesses.
\end{abstract}

Keywords: entrepreneurship; COVID-19; economic crisis; challenges; policies to promote entrepreneurship JEL Classification: I18; L21; L23; L26 


\section{Introducción}

La pandemia actual ha provocado una crisis mundial sin precedentes. Gobiernos, investigadores y ciudadanos de todo el mundo están luchando para responder a la propagación del SARS-CoV-2, el virus que causa la enfermedad por coronavirus 2019 (COVID-19). En el momento de este artículo, la COVID-19 ya ha superado los 8 millones de casos en todo el mundo y ha derivado en la muerte de más de 440.000 personas.

Más allá de la tragedia humanitaria, el virus también está teniendo un impacto local y global en las economías de todo el mundo. Los efectos, no sólo del confinamiento, sino de los temores y preocupaciones que rodean los efectos imprevisibles de la COVID-19 pronostican una recesión que amenaza el funcionamiento y desempeño de las empresas.

Los empresarios no siempre están preparados para afrontar periodos de crisis económicas, pero sin duda nadie se había preparado para los eventos de los últimos meses. Esto ha producido una paralización de gran parte de la actividad debido a los cierres temporales o definitivos de la actividad y los descensos de la demanda, generando a su vez una alta incertidumbre y una gran perdida de capital humano.

Este trabajo surge de la necesidad de conocer como esta singular situación, nunca vivida hasta el momento, afecta a los emprendedores en su actividad empresarial diaria. Para ello, esta investigación parte del análisis de la literatura sobre emprendimiento y crisis económica con la cual se pretende conocer cuál es el papel de los emprendedores ante una situación de crisis económica, así como saber cuales son los principales desafíos a los que se tienen que enfrentar para crear o mantener su empresa en este tipo de situaciones. Tras la revisión de la literatura, se realiza un análisis cuantitativo sobre una muestra de 236 emprendedores españoles, ubicados geográficamente en la Región de Murcia. Este análisis pone de manifiesto las diversas incertidumbres y desafíos a los que se tienen que enfrentar los emprendedores en esta crisis, que no tienen porqué ser los mismos que en crisis anteriores y, además, se observan como se piensan hacer frente a los mismos.

\section{Emprendimiento y crisis económica}

\subsection{Emprender en tiempos de crisis}

En la década de los años noventa, la burbuja inmobiliaria de Japón y el incremento del precio del petróleo produjo un aumento constante y generalizado de los precios en gran número de países. España fue uno de los países a los que también le afectaron estos hechos, pero todo parece indicar que llegaron con un poco de retraso, o por lo menos, esa fue la sensación que tienen los investigadores. En concreto, existen autores que llegan a afirmar que, en verdad, España se encontraba inmersa en el comienzo de una crisis encubierta por los grandes eventos como fueron la Exposición Universal de Sevilla y los Juegos Olímpicos de Barcelona que se llevaron a cabo a principios de dicha década. Es más, esta situación vivida en España se llega a comparar con la que ocurrió hace apenas unos años con los mundiales celebrados en Brasil en el año 2014 (Muñoz Palacios, 2017).

Precisamente, se produjo un gran incremento del desempleo tras la finalización de estos eventos, lo que se provocó un gran descenso en las cifras económicas de nuestro país. Así, el PIB llegó a reducirse en un 1,1\% en el segundo trimestre de 1992, manteniendo resultados negativos hasta el tercer trimestre de 1993, cuando esta consiguió crecer un 0,9\%. Estos datos de principios de los noventa provocaron que el gobierno español optara por realizar un gran esfuerzo inversor, fomentando el crecimiento de la economía aunque a costa de unos altos niveles de endeudamiento público.

A pesar de este esfuerzo por parte del gobierno español, el mayor dato sobre desempleo en esta crisis se produjo en el año 1994, llegando a alcanzar la cifra de 24,1\% (información similar a las tasas registradas en la crisis del año 2008, aunque se debe destacar que esta crisis de principios de los noventa fue intensa, pero de menor duración). Fue precisamente a partir de ese momento, ya en 1995, cuando se puede empezar a hablar de recuperación económica, al obtener unos datos de 400.000 puestos de trabajados creados, lo que provocó un descenso de la tasa de desempleo de más de dos puntos porcentuales.

Sin embargo, en 1996, los informes ofrecidos por el Ministerio de Trabajo (2009) indicaban que el Estado continuaba teniendo pérdidas, a pesar de que el PIB continuaba en aumento y que la creación de puestos de trabajo había ascendido a la cifra de 500.000. Pero, no fue hasta 1997, gracias a la entrada en vigor del Pacto de Toledo, con el cual se fundó el Fondo de Reserva de Seguridad Social, cuando se consiguió la subsanación de las cuentas de la Seguridad Social española.

Precisamente, fue en este momento, en 1997, cuando se afirma que, realmente, empiezan los años de prosperidad económica y creación de empleo principalmente en el sector de la construcción. Este sector económico fue considerado a principios de siglo como el principal motor de la economía española e, ironías del 
destino, supuso también una de las principales causas de la siguiente crisis ocurrida en nuestro país, la del año 2008 (Muñoz Palacios, 2017).

Pero no solo el sector de la construcción en España fue origen de la crisis de 2008. Esta tuvo su origen en los Estados Unidos y se propagó con rapidez a escala mundial. Entre las causas de su origen se mencionan factores como los altos precios de las materias primas, la sobre valorización del producto, una crisis alimentaría mundial, una elevada inflación planetaria y la amenaza de una recesión en todo el mundo, así como una crisis crediticia, hipotecaria y de confianza en los mercados (Peñaherrera León \& Cobos Alvarado, 2012). Esta crisis trajo consigo grandes retos a los que tuvieron que hacer frente las diferentes economías: las nuevas reformas laborales, la necesidad de aumento de productividad empresarial, la recuperación de puestos de trabajo, encontrar nuevos nichos y vías de negocios, las tecnologías de la información y la comunicación, el proceso de globalización, la creciente competencia en los mercados, la aparición de mercados emergentes.

España buscó soluciones, como medidas anti-crisis, en el sistema empresarial de pequeñas y medianas empresas (Peñaherrera León \& Cobos Alvarado, 2012). La recuperación de esta profunda y reciente crisis se produjo en diferentes estadios de tiempo según el territorio que se analiza. En el caso español, también existen diferencias teniendo en cuenta las distintas comunidades autónomas. Así, Ortega (2019) indica que en España se tardó entre 6 y 9 años en recuperar el PIB per cápita, y que fueron las comunidades de Cantabria, Asturias, Castilla-La Mancha, Valencia, Murcia y Andalucía las que no lo hicieron hasta 2017.

Ya en la actualidad, año 2020, nos encontramos en un periodo en el que la mayor preocupación de la población española es que la crisis sanitaria de la COVID-19 se convierta en otro periodo de recesión económica, al tener tan presente la profunda y larga crisis del 2008. Lo cierto es que, aunque apenas ha transcurrido el tiempo, ya se pronostican una gran desaceleración económica este año que provocará un descenso del PIB del 9\% y un incremento de medio millón en el número de parados (CEOE, 2020). Por lo que, todo parece indicar que nos encontramos en el inicio de otro período de crisis económica que ya está tenido consecuencias devastadoras para muchas economías.

Pese a que el término crisis económica se asemeja a aspectos negativos y de dificultad económica y social, algunos autores van más allá del pensamiento económico clásico (según el cual se esperaría que cuando el crecimiento de la economía es bajo existan menos personas involucradas en una actividad emprendedora pues hay menos oportunidades de negocio) para argumentar que, cuando surgen crisis económicas, el número de personas involucradas en la actividad emprendedora tiende a aumentar (Morcillo, 2005). Es precisamente entonces cuando aparece el autoempleo y el emprendimiento como verdadera palanca de cambio para la recuperación económica y la creación de empleo (Jiménez Zarco, 2012).

El emprendimiento es relevante porque sirve como un mecanismo que ayuda a procesar las crisis económicas, al reubicar recursos de tal manera que prometen nuevas actividades que reemplazan a las que están obsoletas (Parra Miranda, 2014). El emprendimiento favorece la creación de nuevos puestos de trabajo, la introducción de nuevos productos en el mercado y genera competencia. La creación de una nueva empresa permite acceder a nuevas oportunidades laborales e incluso ofrece la oportunidad de percibir ganancias salariales superiores a las del trabajo por cuenta ajena (Constant \& Zimmermann, 2006).

Por ello, sobre la base de estos argumentos, tanto desde el ámbito científico como desde las instituciones económicas nacionales e internacionales cada vez más se trata de fomentar el emprendimiento, y de forma especial en situaciones de crisis como la originada por razón de la COVID-19. Las medidas de generación y promoción de nuevas iniciativas emprendedoras y de apoyo a los autónomos y emprendedores ya existentes surgen con el objetivo de convertir a este colectivo en la palanca de cambio de la salida de la crisis y de la creación de empleo cuando se den las condiciones. El emprendedor actúa como un instrumento para dinamizar las economías y promover la flexibilidad del tejido productivo a través de la creatividad y la innovación, junto con otras políticas de fortalecimiento económico generando cambios estructurales en la economía local, regional y nacional (Peñaherrera León \& Cobos Alvarado, 2012).

\subsection{Emprendimiento y perfil emprendedor}

A pesar de la popularidad de la que goza en la actualidad la figura del empresario, en los estudios sobre el crecimiento y desarrollo económico no siempre ha merecido tanta atención, a pesar de que ya en el trabajo de Cantillon (1978), precursor directo de Adam Smith, sí se le concede un papel muy relevante a este personaje. Sin embargo, hay que señalar que, a pesar de ese olvido del grueso de las ciencias de la figura del empresario, sí han existido autores que han puesto de manifiesto la importancia de su papel. De entre ellos, sin duda, el economista más destacado es Schumpeter (1911), quien ideó la teoría del Desarrollo Económico basada en la actuación innovadora del empresario (Liñán, 2004).

Muchas de las aportaciones posteriores en este campo se basan, de una forma o de otra, en la del profesor Schumpeter, cuyo trabajo tiene sin duda un carácter seminal dentro de los estudios de la función empresarial. Así, por ejemplo, Leibenstein (1968) distingue dos grandes tipos de actividad empresarial, en un extremo 
estaría la actividad empresarial rutinaria, que es en realidad una especie de gestión, y en el resto del espectro se encontraría la actividad empresarial Schumpeteriana o 'de nuevo tipo'.

Precisamente, la importancia de la figura del emprendedor ante una crisis económica, así como la distinción entre tipos de actividad empresarial han provocado una mayor investigación estos últimos años sobre la persona que decide poner en marcha la empresa. Existen determinadas características individuales que se encuentran en un emprendedor: confianza (debe creer en su idea, la cual convierte en un proyecto y este, a su vez, en una realidad); fortaleza (se considera indispensable en el emprendedor de éxito, ya que no se debe rendir y, si no sale bien esa aventura, debe levantarse con más fuerza para afrontar la siguiente); innovación (para conseguir diferenciarse de la competencia y alcanzar las exigencias del consumidor, haciendo de su proyecto algo diferente) y faceta comercial (debe tener competencia comercial para vender el producto o servicio desarrollado) (Muñoz Palacios, 2017).

Pero no solo estas características, el perfil del emprendedor, en general, ha sido analizado y, es más, existen investigaciones que afirman que este difiere si se atiende a un periodo de auge o de crisis económica. Así, por ejemplo, observando los datos ofrecidos al respecto por el proyecto GEM (Gutiérrez Solana et al., 2018) en estos años en los que se sufrió la crisis iniciada en el 2008, se comprueba, por ejemplo, que es mayor la proporción de individuos que emprenden por necesidad (quizá porque estos han perdido su empleo o porque han reducido sus ingresos en el ámbito laboral), también aumenta la edad media de los emprendedores (está demostrado que a mayor edad disminuye la inserción laboral de las personas, por lo que, estas pueden optar por crear una empresa al no encontrar trabajo en el mercado laboral), o el cambio del sector de actividad elegido a la hora de poner en marcha su empresa.

En concreto, sobre el sector de actividad, fue el industrial el que en menor medida fue escogido por los emprendedores para crear su empresa, quizá porque en él se encuentra incluido el sector de la construcción, pero no solo por este motivo, también por la menor o casi inexistente inversión en I+D+i y la fuga de cerebros que provocaron el apalancamiento de este sector. En su lugar, los emprendedores se decantaron por el sector servicios (principalmente por el modelo de éxito probado, como es considerada la franquicia, que hacía más atractivo a este sector), pero, en realidad, se debe destacar que, en mayor medida, los individuos escogieron el sector primario (debido principalmente a los recursos naturales que posee España, así como por a la importante inversión en ayudas al emprendimiento y sostenimiento del sector agrícola ofrecido por la Política Comunitaria Agraria) (Muñoz Palacios, 2017).

Por lo tanto, a modo de resumen se puede decir que los emprendedores han sido, son y serán un recurso fundamental en periodos de crisis, en los que se dan oportunidades para renovar la economía de un territorio a través de su papel. De ahí que, ante esta crisis provocada por la COVID-19, lo que se tiene que hacer sea promover la investigación en emprendimiento con el fin de tener la información necesaria con la que desarrollar políticas eficientes en nuestro país que promuevan este necesario fenómeno empresarial, el cual provocará la regeneración económica del país y, por tanto, la salida de la crisis.

\subsection{Desafíos del emprendimiento}

\section{Digitalización}

Antes de la crisis de la COVID-19, la digitalización en las empresas ya era una clave competitiva que permitía no sólo una comunicación más fluida, una mayor eficiencia de las operaciones internas, sino también el acceso a un mercado abierto y competitivo. La digitalización, además de generar autonomía y aumentar el aprendizaje colectivo, entender mejor el entorno y, por tanto, adaptarse y aprovechar mejor las oportunidades que este ofrece, a su vez, facilita la planificación, impulsa el desarrollo de nuevos productos, servicios y procesos.

Pero, fue precisamente con la crisis sanitaria sufrida cuando se impuso el distanciamiento físico entre personas lo que limitó la capacidad de reunirse e interactuar. Por este motivo, los empresarios han encontrando formas creativas de mantener y fomentar la conexión entre individuos mientras están físicamente separados. Así, las redes sociales como Twitter, Instagram y TikTok y las aplicaciones de videoconferencia como Google Meet, Webex, Zoom y Kudo han demostrado ser esenciales durante este período para que muchas organizaciones pudieran ofrecer sus productos o servicios. Además, varias compañías tecnológicas importantes como Cisco (la compañía detrás del servicio de conferencia web Webex), Google, Facebook, Apple y Amazon están donando recursos para los sistemas educativos y de atención médica y expandiendo el acceso gratuito a sus productos y programas de servicio al cliente (Haeffele, Hobson, \& Storr, 2020).

Precisamente, gracias a estos servicios se han podido ofrecer los productos o servicios de manera diferente, al no poderse realizar de manera presencial. Como ejemplo de ello se pueden encontrar los gimnasios que ofrecían clases virtuales, la realización de conciertos virtuales, visitas a museos y otras experiencias como los artistas que también están alentando a otros a involucrarse en el arte a través de medios telemáticos. Las diversas formas en que los individuos satisfacen las necesidades son demasiado numerosas para enumerarlas 
por completo, pero está claro que a través de la digitalización ha sido posible satisfacer las mismas en el periodo de confinamiento vivido (Haeffele et al., 2020).

\section{Financiación}

La rápida propagación de la COVID-19 ha tenido un impacto dramático en los mercados financieros de todo el mundo, creando un nivel de riesgo sin precedentes, lo que provocó que los inversores sufrieran importantes pérdidas en muy poco tiempo. Precisamente, el brote ha tenido claros impactos económicos significativos. En el corto plazo, como muchos países adoptaron estrictas políticas de cuarentena, sus actividades económicas se vieron significativamente limitadas. A largo plazo, las consecuencias de esta pandemia pueden ocasionar unos niveles elevados desempleo y gran número de quiebras comerciales. Algunas industrias, como el turismo y la aviación con certeza se puede afirmar que tendrán grandes dificultades (Zhang, Hu, \& Ji, 2020).

En este punto es preciso señalar que, si bien los impactos económicos globales exactos aún no están claros, los mercados financieros ya han respondido con movimientos dramáticos. En marzo de 2020, ante los desplomes de mercados de valores como el de EE.UU., Europa, Asia, Reino Unido y Japón los bancos centrales y las autoridades respondieron de inmediato desarrollando instrumentos como la Reserva Federal (FED), por la que se anunció un interés del cero por ciento en la política de tarifas y al menos un programa de flexibilización cuantitativa (QE) de 700 mil millones de dólares. Siguiendo las respuestas negativas a esta política en el mercado, la FED anunció una política de QE ilimitada ocho días después. Aunque la mayoría de los mercados bursátiles han comenzado recientemente a recuperarse, un gran número de ellos sigue encontrándose en situación de incertidumbre mientras no sea erradicada la pandemia (Zhang et al., 2020).

Si se atiende al caso español, el Gobierno ha desarrollado un programa centrado hasta la fecha en garantizar medidas de liquidez para sostener el tejido productivo y programas de protección para los más vulnerables. Precisamente, las medidas parten de la idea de que para mitigar tanto la crisis económica como social que se avecina será imprescindible simplificar los trámites para la concesión de las ayudas, la ampliación de su alcance en determinados casos y la adopción de medidas que permitan la adaptación a la recuperación gradual de la actividad (CEOE, 2020).

Es más, se considera que las acciones necesarias son de gran calado y han de mantenerse en el tiempo, ya que los bancos, precisamente por su naturaleza, no son vulnerables en tiempos de recesiones económicas, debido a la probabilidad de préstamos morosos y la posibilidad en casos extremos de corridas bancarias, de ahí que no puedan ser considerados como principal fuente de financiación en este tipo de periodo económico (Goodell, 2020). De ahí que se afirme que para dar credibilidad a este programa se requiere de un amplio consenso político apoyado por los empresarios y los interlocutores sociales. Para ello, resulta imprescindible la elaboración de un plan de financiación relevante basado en la colaboración público-privada, en las que se recojan medidas en el ámbito tributario y un decidido y firme apoyo financiero por parte de la Unión Europea (CEOE, 2020).

\section{Percepción de oportunidades}

Percibir oportunidades es un proceso que depende directamente de la capacidad del individuo para detectar brechas inexploradas o nichos bajo procesos dinámicos, creativos y reflexivos (Baron, 2004; Zander, 2004). Los emprendedores, a menudo, se caracterizan por una fuerte intuición que identifica oportunidades negocios rentables y generalmente apoyan sus ideas en anticipación de otros actores del mercado (Baron, 2004; Nicolás Martínez, Rubio Bañón, \& Fernández Laviada, 2019). Así, siguiendo a Schumpeter (1911), se debe tener en cuenta que los periodos en los que es posible encontrar un mayor número de oportunidades es en las épocas de crisis como la que se está viviendo.

Precisamente, el argumento de Schumpeter (1911) es hoy en día destacado con mayor fuerza. Esto es así porque, a pesar de la respuesta desarrollada por el Gobierno para conseguir una rápida recuperación, la experiencia respecto a desastres en años anteriores pone de manifiesto que los gobiernos pueden no comprender adecuadamente el alcance de la crisis y, por lo tanto, no movilizar recursos necesarios. Se considera este hecho debido a que es estructuralmente difícil para las burocracias reorganizar y desplegar servicios sociales nacionales para ayudar con las circunstancias que cambian rápidamente, por ejemplo, proporcionando pruebas y suministros médicos, comidas a personas mayores y escolares en sus hogares, etc. (Haeffele et al., 2020).

Por el contrario, los empresarios ven estos problemas de coordinación como oportunidades, siendo más capaces de adaptarse a necesidades y circunstancias cambiantes que los organismos públicos. Para facilitar el emprendimiento, en respuesta a la pandemia de la COVID-19, los encargados de formular políticas deben permitir a los empresarios experimentar para encontrar soluciones para los problemas emergentes debido a la actual situación. Esto significa que eliminar las barreras al emprendimiento debe ser una actividad necesaria en cualquier agenda política. Así, por ejemplo, lo comentado se puede observar cuando muchas empresas e 
industrias que fabrican productos que pueden no ser de vital importancia han cambiado su proceso productivo y, en la actualidad, producen suministros médicos y de limpieza cruciales para evitar la propagación de la pandemia (Haeffele et al., 2020).

\section{Capital humano}

La correcta gestión organizacional es una de las prácticas empresariales más estudiada y analizada debido a la importancia que esta tiene para su eficiente funcionamiento. A la hora de buscar ventajas competitivas en una organización, existen investigaciones que se van fijando en los diferentes elementos que forman la empresa como posibles fuentes. Pero, no tan recientemente, en una corriente se afirma que la principal fuente de ventaja competitiva es aquella que se basa en sus elementos intangibles, ya que como indica la teoría de recursos y capacidades, ellos son valiosos, raros, difíciles de imitar y de sustituir (Nicolás \& Rubio, 2015).

Precisamente, por los argumentos encontrados en esta teoría a la hora de gestionar eficientemente una organización, los investigadores centran su atención en la correcta gestión de sus recursos humanos. Estos elementos, a su vez, toman mayor importancia cuando la organización no fabrica un producto, sino que ofrece un servicio, debido a que son sus recursos humanos lo que deben realizar el mismo para cada cliente día a día y deben intentar dar el mejor servicio a todos ellos (Nicolás \& Rubio, 2015).

Con la pandemia de la COVID-19, a medida que el virus se propagó rápidamente desde Europa y Asia al resto del mundo, los hospitales se convirtieron rápidamente en zonas calientes para el tratamiento y la transmisión de esta enfermedad. Todo esto dio lugar a que gran número de trabajadores sanitarios se contagiasen con esta enfermedad, lo que provocó que disminuyeran los recursos humanos disponibles para atender a la población. Por lo que, esta crisis sanitaria mostró que la existencia de recursos limitados y que el personal bajo presión incrementa de manera considerable los riesgos de transmisión y la carga sobre nuestros sistemas sanitarios (Brindle \& Gawande, 2020).

Pero si se está atendiendo a los recursos humanos, no se debe pensar que lo que ocurrió en el ámbito sanitario fue algo generalizado en el resto de sectores. En este punto se debe recordar que el confinamiento con la situación del estado de alarma provocó que en España se paralizara casi la totalidad de los sectores económicos (a excepción de los que se dedicaban a servicios básicos, como puede ser la alimentación). Como se puede esperar, la mayoría de empresas no podían soportar los costes acarreados por tener la empresa cerrada durante semanas, lo que provocó que pidieran ERTEs con los que limitar los costes salariales tanto si la empresa había cerrado total o parcialmente. Como era de esperar, gran número de empresas solicitaron ERTEs, lo que provocó un colapso en la administración, la cual tuvo que dar por aprobados muchos de los mismos por silencio administrativo, lo que, a su vez, provocó que miles de trabajadores se encontraran en situación de ERTE durante el confinamiento (CEOE, 2020).

En este punto, cabe destacar que no solamente existieron medidas para los trabajadores, además, se aprobó en el RD 15/2020, del 21 de abril, medidas para ayudar a los trabajadores autónomos confinados por la COVID-19 ante la falta de ingresos. Hoy en día los trabajadores están volviendo progresivamente a sus puestos de trabajo después de estar incluidos o no en un ERTE, aunque se debe decir que existe gran cantidad de empresas que cerraron por el confinamiento y que, parece, que no van a volver a abrir sus puertas.

\section{Metodología y resultados}

\subsection{Muestra, obtención de información y variables}

La información se ha obtenido a través de la respuesta al cuestionario elaborado por la Red GEM España (Observatorio del Emprendimiento) de la que forma parte la Universidad de Murcia. El cuestionario se envió entre los días 20 y 21 de abril a emprendedores de la Región de Murcia por correo electrónico y se difundió a través de diversas redes sociales, con el apoyo de agentes del ecosistema emprendedor de la Región de Murcia. Se mantuvo abierto hasta el día 30 de abril de 2020. Contestaron un total de 236 empresas en la Región de Murcia.

A la hora de analizar estadísticamente las diferencias de comportamiento en las variables objeto de estudio, y considerando los factores antigüedad, existencia de trabajadores por cuenta ajena, género y nivel educativo del gerente se utilizará el análisis de tablas de contingencia aplicando el test de la $\mathrm{X}^{2}$ de Pearson con el fin de valorar si dos variables se encuentran relacionadas. Todas las variables utilizan medidas de percepción cualitativa, basada en la opinión que tiene el directivo con respecto a una serie de afirmaciones o escalas, tal y como se muestran en la Table 1. 
Table 1. Medidas de las variables analizadas

\section{VARIABLES}

Situación actual de la actividad Señale UNA opción 1: Continúa operando normalmente

que describa el efecto COVID 19 sobre la situación

actual de su actividad

2: Continúa operando con teletrabajo

3: He cerrado temporalmente por decisión administrativa

4: He cerrado temporal o definitivamente

Situación actual de la demanda ¿Cómo se ha visto afectada la demanda de sus productos/servicios durante la situación de confinamiento? (señale UNA opción)

Situación de la plantilla En caso de tener

1: No tengo demanda (negocio cerrado)

2: Se ha reducido notablemente

3: Ha aumentado o se ha mantenido constante

trabajadores contratados ¿Ha tomado alguna medida

excepcional en materia laboral debido a esta situación (señale UNA opción)

2: Expedientes de regulación (ERTE, ERE)

3: Despidos

4: Ambas medidas (expedientes de regulación y despido)

Preocupaciones ¿Cuál de las siguientes dificultades considera que serán claves para los nuevos emprendedores en los meses siguientes? (puede indicar VARIAS opciones)

1: No superar un periodo de aislamiento tan largo

2: No poder mantener a los empleados

3: No poder contratar empleados

4: No se atreverán a asumir riesgos

5: Falta de financiación privada

6: No habrá fondos públicos suficientes

7: Falta de formación en sectores líderes para el futuro

8: Desánimo y falta de interés del personal

9: Falta de interés de las administraciones por dar impulso

10: Se cierran fronteras y ello perjudica a emprendedores

Digitalización: Si está realizando teletrabajo ¿Qué medios tecnológicos ha empleado en mayor medida?

1: Videoconferencias

2: Plataformas para compartir archivos

3: Red interna virtual (VPN)

4: Misma tecnología que el puesto presencial

Financiación Pública: ¿Cómo es su estado respecto a 1: Estoy recibiendo ayudas públicas

la recepción de ayudas públicas por el COVID 19?

(señale UNA opción)

2: Estoy pendiente de respuesta

3: Me han denegado

4: No he solicitado

Oportunidades: La situación actual, ¿puede propiciar 1: Sí, para cualquier empresa

que aparezcan nuevas oportunidades en el mercado? 2: Sí, para empresas con recursos suficientes y orientadas a actividades

relacionadas con la resolución de la crisis

3: Sí, para empresas con recursos suficientes y diversificadas

4: No o excepcionalmente, pero no con carácter general

Tipo de iniciativas

0: Nuevas iniciativas (- 42 meses de actividad)

1: Iniciativas consolidadas (+ 42 meses de actividad)

Trabajadores contratados

0: $\mathrm{NO}$

1: SI

Género del gerente

0: Mujer

1: Hombre

Estudios del gerente

0: Sin estudios, primarios, ESO, FP.

1: Universitarios

Fuente: Elaboración propia

\subsection{Resultados: situación actual}

Los datos analizados (Table 2) reflejan una fuerte caída de la actividad presencial en las organizaciones que ha disminuido en un 86,9\% durante las primeras semanas del estado de alarma. El 46,2\%, ha podido adoptar medidas organizativas que evitan situaciones de contacto social y otro $26,7 \%$ ha tenido que cerrar temporalmente por decisión administrativa. El 14\% restante ha cerrado por decisión propia, temporal (11,9\%) o definitivamente $(3,1 \%)$

Un análisis en función del tipo de iniciativa, trabajadores contratados por cuenta ajena, género y nivel de estudios, muestra diferencias significativas para cada uno de los grupos. Concretamente, las empresas que siguen operando normalmente $(13,1 \%)$, bien sea presencialmente o a través de teletrabajo, se caracterizan por ser empresas consolidadas, al llevar operando más de 42 meses, por tener trabajadores contratados y estar dirigidas por hombres con estudios universitarios. Las diferencias son especialmente marcadas en el caso del teletrabajo.

Las empresas más afectadas por los cierres son, según los datos de la Table 2, las de menos antigüedad, las que no tienen trabajadores contratados y aquellas cuyos gerentes no tienen estudios universitarios. Los cierres, temporales o definitivos, han afectado más a las empresas dirigidas por hombres. 
Table 2. Situación actual de su actividad

\begin{tabular}{|c|c|c|c|c|c|c|c|c|c|}
\hline \multirow[b]{2}{*}{ SITIUACIÓN ACTUAL } & \multirow[b]{2}{*}{ Total } & \multicolumn{2}{|c|}{ Tipo de iniciativa } & \multicolumn{2}{|c|}{$\begin{array}{l}\text { Trabajadores } \\
\text { contratados }\end{array}$} & \multicolumn{2}{|c|}{ Género } & \multicolumn{2}{|c|}{ Nivel estudios } \\
\hline & & $\begin{array}{l}\text { Nuevas } \\
\text { inic. }\end{array}$ & $\begin{array}{l}\text { Inic. } \\
\text { cons. }\end{array}$ & NO & SI & Hombre & Mujer & No univ. & Univ. \\
\hline Continúa operando normalmente & $13,1 \%$ & $11,8 \% * * *$ & $14,4 \% * * *$ & $10,2 \% *$ & $15,7 \% *$ & $13,8 \% * * *$ & $11,8 \% * * *$ & $12,7 \% * * *$ & $13,3 \% * * *$ \\
\hline Continúa operando con teletrabajo & $46,2 \%$ & $38,2 \% * * *$ & $53,4 \% * * *$ & $42,4 \% *$ & $51,0 \% *$ & $51,0 \% * * *$ & $36,8 \% * * *$ & $21,8 \% * * *$ & $54,5 \% * * *$ \\
\hline $\begin{array}{l}\text { He cerrado temporalmente por } \\
\text { decisión administrativa }\end{array}$ & $26,7 \%$ & $34,3 \% * * *$ & $19,5 \% * * *$ & $28,8 \% *$ & $24,5 \% *$ & $19,3 \% * * *$ & $40,8 \% * * *$ & $40,0 \% * * *$ & $21,8 \% * * *$ \\
\hline He cerrado temporal o definitivamente & $14,0 \%$ & $15,7 \% * * *$ & $12,7 \% * * *$ & $18,6 \% *$ & $8,8 \% *$ & $15,9 \%$ *** & $10,5 \%$ \%** & $25,5 \% * * *$ & $10,3 \%$ *** \\
\hline Total & $100,0 \%$ & $46,4 \%$ & $53,6 \%$ & $54,0 \%$ & $46,0 \%$ & $64,4 \%$ & $35,6 \%$ & $25,0 \%$ & $75,0 \%$ \\
\hline
\end{tabular}

Fuente: Elaboración propia

p-valor: $(*): \mathrm{p}<0.1 ;(* *): \mathrm{p}<0.05 ;(* * *): \mathrm{p}<0.01$

El análisis de los efectos de la pandemia en la demanda de productos y servicios (Table 3) muestra que el $50,2 \%$ de empresas han visto reducida su demanda notablemente, mientras que el $30 \%$ ha visto paralizada su actividad al tener sus negocios cerrados. El 19,8\% restante han incrementado su demanda o no han visto afectada la cantidad de productos o servicios vendidos. No existen diferencias significativas con el resto de variables analizadas.

Table 3. Situación actual de la demanda

\begin{tabular}{|c|c|c|c|c|c|c|c|c|c|}
\hline \multirow[b]{2}{*}{ SITIUACIÓN ACTUAL } & \multirow[b]{2}{*}{ Total } & \multicolumn{2}{|c|}{ Tipo de iniciativa } & \multicolumn{2}{|c|}{$\begin{array}{l}\text { Trabajadores } \\
\text { contratados }\end{array}$} & \multicolumn{2}{|c|}{ Género } & \multicolumn{2}{|c|}{ Nivel estudios } \\
\hline & & $\begin{array}{l}\text { Nuevas } \\
\text { inic. }\end{array}$ & $\begin{array}{l}\text { Inic. } \\
\text { cons. }\end{array}$ & NO & SI & Hombre & Mujer & No univ. & Univ. \\
\hline No tengo demanda (negocio cerrado) & $30,0 \%$ & $32,3 \%$ & $30,4 \%$ & $29,8 \%$ & $31,1 \%$ & $30,3 \%$ & $30,8 \%$ & $30,9 \%$ & $30,4 \%$ \\
\hline Se ha reducido notablemente & $50,2 \%$ & $48,4 \%$ & $50,7 \%$ & $51,6 \%$ & $50,5 \%$ & $50,9 \%$ & $50,0 \%$ & $47,3 \%$ & $50,7 \%$ \\
\hline $\begin{array}{l}\text { Ha aumentado o se ha mantenido } \\
\text { constante }\end{array}$ & $19,8 \%$ & $19,4 \%$ & $18,9 \%$ & $18,5 \%$ & $18,4 \%$ & $18,9 \%$ & $19,2 \%$ & $21,8 \%$ & $18,9 \%$ \\
\hline Total & $100,0 \%$ & $46,4 \%$ & $53,6 \%$ & $54,0 \%$ & $46,0 \%$ & $64,4 \%$ & $35,6 \%$ & $25,0 \%$ & $75,0 \%$ \\
\hline
\end{tabular}

Fuente: Elaboración propia

p-valor: $(*): \mathrm{p}<0.1 ;(* *): \mathrm{p}<0.05 ;(* * *): \mathrm{p}<0.01$

Las situaciones expuestas anteriormente tienen su reflejo en los empleados por cuenta ajena, tal y como muestra la Table 4. Si bien hay un 46,3\% de actividades que aseguran seguir operando con normalidad, se observa que hay un $14,8 \%$ de emprendedores que han optado por realizar ERTEs o ERES, un 25,1\% han optado por despedir o extinguir los contratos de sus trabajadores y un 13,8\% han adoptado ambas medidas.

Un análisis de las variables antigüedad, género y nivel de estudios del gerente muestra que sólo existen diferencias significativas para la primera de las variables. Concretamente, las iniciativas nuevas son las que han visto menos afectadas sus plantillas con despidos o expedientes de regulación de empleo.

Table 4. Situación actual de la plantila

\begin{tabular}{|c|c|c|c|c|c|c|c|}
\hline \multirow{3}{*}{\begin{tabular}{|l} 
SITIUACIÓN ACTUAL \\
No, sigue con normalidad \\
\end{tabular}} & \multirow{3}{*}{$\begin{array}{l}\text { Total } \\
46,3 \%\end{array}$} & \multicolumn{2}{|c|}{ Tipo de iniciativa } & \multicolumn{2}{|c|}{ Género } & \multicolumn{2}{|c|}{ Nivel estudios } \\
\hline & & $\begin{array}{c}\text { Nuevas } \\
\text { inic. }\end{array}$ & Inic. cons. & Hombre & Mujer & No univ. & Univ. \\
\hline & & $54,9 \% * * *$ & $39,6 \% * * *$ & $44,4 \%$ & $50,0 \%$ & $50,0 \%$ & $45,2 \%$ \\
\hline Expediente de regulación & $14,8 \%$ & $20,9 \% * * *$ & $9,9 \% * * *$ & $13,5 \%$ & $17,1 \%$ & $10,7 \%$ & $16,4 \%$ \\
\hline Despidos & $25,1 \%$ & $16,5 \% * * *$ & $31,5 \% * * *$ & $28,6 \%$ & $18,6 \%$ & $26,8 \%$ & $24,0 \%$ \\
\hline Ambas medidas & $13,8 \%$ & $7,7 \% * * *$ & $18,9 \% * * *$ & $13,5 \%$ & $14,3 \%$ & $12,5 \%$ & $14,4 \%$ \\
\hline Total & $100,0 \%$ & $46,4 \%$ & $53,6 \%$ & $64,4 \%$ & $35,6 \%$ & $25,0 \%$ & $75,0 \%$ \\
\hline
\end{tabular}

Fuente: Elaboración propia

p-valor: $(*): p<0.1 ;(* *): p<0.05 ;(* * *): p<0.01$

Respecto a los miedos y dificultades derivados de la emergencia sanitaria, el temor a no superar un periodo tan largo de aislamiento se encuentra a la cabeza de las preocupaciones de los emprendedores, manifestado así por el 67,8\% de los mismos -Table 5-, seguido por el miedo a asumir riesgos (57,2\%). A continuación, los emprendedores temen no obtener los fondos públicos suficientes $(53,4 \%)$. Le sigue en importancia la preocupación por el desánimo personal $(35,2 \%)$ y la falta de interés de las administraciones por dar impulso a las actividades empresariales (31.8\%). La preocupación por no poder mantener el empleo actual (28,8\%), el miedo al cierre de fronteras $(26,7 \%)$, la falta de financiación de los proyectos $(22,9 \%)$ y de formación en sectores líderes $(22,0 \%)$ son los siguientes en la lista. No poder contratar nuevos empleados se sitúa con un $13,6 \%$ al final del ranking. 
Table 5. Situación actual preocupaciones

\begin{tabular}{|c|c|c|c|c|c|c|c|c|c|}
\hline \multirow[b]{2}{*}{ SITIUACIÓN ACTUAL } & \multirow[b]{2}{*}{ Total } & \multicolumn{2}{|c|}{ Tipo de iniciativa } & \multicolumn{2}{|c|}{$\begin{array}{l}\text { Trabajadores } \\
\text { contratados }\end{array}$} & \multicolumn{2}{|c|}{ Género } & \multicolumn{2}{|c|}{ Nivel estudios } \\
\hline & & $\begin{array}{l}\text { Nuevas } \\
\text { inic. }\end{array}$ & $\begin{array}{l}\text { Inic. } \\
\text { cons. }\end{array}$ & NO & SI & Hombre & Mujer & No univ. & Univ. \\
\hline $\begin{array}{l}\text { No superar un periodo de aislamiento } \\
\text { tan largo }\end{array}$ & $67,8 \%$ & $66,1 \%$ & $69,0 \%$ & $70,9 \%$ & $64,8 \%$ & $66,4 \%$ & $70,2 \%$ & $76,2 \% *$ & $64,5 \% *$ \\
\hline No poder mantener a los empleados & $28,8 \%$ & $14,7 \% * * *$ & $40,5 \% * * *$ & $1,6 \% * * *$ & $61,1 \% * * *$ & $33,6 \% * *$ & $20,2 \% * *$ & $25,4 \%$ & $29,7 \%$ \\
\hline No poder contratar empleados & $13,6 \%$ & $16,5 \%$ & $11,1 \%$ & $11,8 \%$ & $15,7 \%$ & $14,5 \%$ & $11,9 \%$ & $9,5 \%$ & $15,1 \%$ \\
\hline No se atreverán a asumir riesgos & $57,2 \%$ & $50,5 \% * *$ & $63,5 \% * *$ & $52,0 \% *$ & $63,0 \% *$ & $55,9 \%$ & $59,5 \%$ & $57,1 \%$ & $57,6 \%$ \\
\hline Falta de financiación privada & $22,9 \%$ & $22,0 \%$ & $23,8 \% * *$ & $17,3 \% * *$ & $29,6 \% * *$ & $25,7 \%$ & $17,9 \%$ & $19,0 \%$ & $24,4 \%$ \\
\hline No habrá fondos públicos suficientes & $53,4 \%$ & $56,0 \%$ & $50,8 \%$ & $55,9 \%$ & $50,9 \%$ & $48,7 \% *$ & $61,9 \% *$ & $52,4 \%$ & $53,5 \%$ \\
\hline $\begin{array}{l}\text { Falta de formación en sectores líderes } \\
\text { para el futuro }\end{array}$ & $22,0 \%$ & $20,2 \%$ & $23,8 \%$ & $21,3 \%$ & $23,1 \%$ & $23,7 \%$ & $19,0 \%$ & $20,6 \%$ & $22,7 \%$ \\
\hline $\begin{array}{l}\text { Desánimo y falta de interés del } \\
\text { personal }\end{array}$ & $35,2 \%$ & $39,4 \%$ & $31,7 \%$ & $37,0 \%$ & $33,3 \%$ & $31,6 \%$ & $41,7 \%$ & $28,6 \%$ & $37,4 \%$ \\
\hline $\begin{array}{l}\text { Falta de interés de las } \\
\text { administraciones por dar impulso }\end{array}$ & $31,8 \%$ & $34,9 \%$ & $29,4 \%$ & $30,7 \%$ & $33,3 \%$ & $35,5 \% *$ & $25,0 \% *$ & $38,1 \%$ & $29,7 \%$ \\
\hline $\begin{array}{l}\text { Se cierran fronteras y ello perjudica a } \\
\text { emprendedores }\end{array}$ & $26,7 \%$ & $24,8 \%$ & $28,6 \%$ & $22,8 \%$ & $31,5 \%$ & $30,3 \% *$ & $20,2 \% *$ & $28,6 \%$ & $26,2 \%$ \\
\hline
\end{tabular}

Fuente: Elaboración propia

p-valor: (*): p<0.1; (**): p <0.05; (***): $p<0.01$

La Table 5 pone de manifiesto las preocupaciones y dificultades por la crisis sanitaria en función del tipo de iniciativa, de la existencia o no de trabajadores por cuenta ajena, el género del entrevistado y el nivel de estudios del gerente. De los resultados se desprende que los emprendedores consolidados ven como mayor dificultad para los nuevos emprendedores el que no se atreverán a asumir riesgos (63,5\%), coincidiendo con lo que opinan los emprendedores que tienen trabajadores contratados por cuenta ajena $(63,0 \%)$, señalando estos últimos, además, la falta de financiación privada (29,6\%). Por último, los hombres ven más que las mujeres que las dificultades para nuevos emprendedores estarán en el cierre de fronteras y dificultades en movilidad $(30,3 \%)$, la falta de interés de las Administraciones en dar impulso (35,5\%), sin embargo son las mujeres quienes muestran mayor preocupación ante la suficiencia de fondos públicos $(61,9 \%)$.

Asimismo, las cifras muestran que tanto las empresas consolidadas como las que tienen empleados por cuenta ajena y las que disponen de un gerente varón son las más preocupadas por las dificultades para mantener el empleo a sus empleados con porcentajes del 40,5\% y del 61,1\% y 33,6\% respectivamente.

Por último, son los gerentes con estudios no universitarios los más preocupados por no superar el periodo de aislamiento.

\subsection{Resultados: desafíos futuros}

Respecto de las claves para afrontar este periodo de dificultades, la utilización de medios tecnológicos es la primera clave. La Table 6 muestra que el 50,8\% de las empresas están utilizado como solución tecnológica la videoconferencia. Así mismo, otra aplicación muy útil, adoptada por el 37,7\% de las empresas que teletrabajan, son las plataformas para compartir archivos remotamente. Por último, el 17,8\% afirma estar utilizando las VPN o Redes Privadas Virtuales.

Table 6. Desafios: digitalización

\begin{tabular}{|c|c|c|c|c|c|c|c|c|c|}
\hline \multirow[b]{2}{*}{ CLAVES } & \multirow[b]{2}{*}{ Total } & \multicolumn{2}{|c|}{ Tipo de iniciativa } & \multicolumn{2}{|c|}{$\begin{array}{l}\text { Trabajadores } \\
\text { contratados }\end{array}$} & \multicolumn{2}{|c|}{ Género } & \multicolumn{2}{|c|}{ Nivel estudios } \\
\hline & & $\begin{array}{l}\text { Nuevas } \\
\text { inic. }\end{array}$ & $\begin{array}{l}\text { Inic. } \\
\text { cons. }\end{array}$ & NO & SI & Hombre & Mujer & No univ. & Univ. \\
\hline Videoconferencias & $50,8 \%$ & $54,1 \%$ & $48,4 \%$ & $48,0 \%$ & $54,6 \%$ & $56,6 \% * *$ & $40,5 \% * *$ & $23,8 \% * * *$ & $61,00 * * *$ \\
\hline Plataformas para compartir archivos & $37,7 \%$ & $40,4 \%$ & $35,7 \%$ & $36,2 \%$ & $39,8 \%$ & $44,7 \% * * *$ & $25,0 \% * * *$ & $19,0 \% * * *$ & $44,8 \% * * *$ \\
\hline Red interna virtual (VPN) & $17,8 \%$ & $3,7 \% * * *$ & $30,2 \% * * *$ & $11,8 \% *$ & $25,0 \% *$ & $21,1 \% *$ & $11,9 \% *$ & $9,5 \% * *$ & $20,9 \% * *$ \\
\hline $\begin{array}{l}\text { Misma tecnología que el puesto } \\
\text { presencial }\end{array}$ & $27,1 \%$ & $20,2 \% * *$ & $33,3 \% * *$ & $19,7 \% * * *$ & $36,1 \% * * *$ & $30,9 \% *$ & $20,2 \% *$ & $22,2 \%$ & $29,1 \%$ \\
\hline
\end{tabular}

Fuente: Elaboración propia

p-valor: $(*): \mathrm{p}<0.1 ;(* *): \mathrm{p}<0.05 ;(* * *): \mathrm{p}<0.01$

Un análisis de los datos anteriores según el tipo de iniciativa, según se cuente con trabajadores contratados y según el género de la persona emprendedora (Table 6), muestra que la formación universitaria y el género, en este caso el masculino, determinan positivamente el grado en el que las empresas han utilizado las tecnologías de información y comunicación. Así mismo, las empresas consolidadas y las que tienen contratados trabajadores por cuenta ajena son las que están utilizando la misma tecnología que cuando se trabajaba presencialmente, así como la red interna virtual (VPN). 
Por lo que respecta a los apoyos recibidos, la Table 7 muestra la situación en cuanto a solicitudes y recepción de ayudas públicas puestas en marcha para hacer frente al impacto económico de la crisis. Concretamente, el $17,4 \%$ de los emprendedores que han respondido el cuestionario aseguran que están recibiendo ayudas públicas por la COVID-19, mientras que sólo a un 6,4\% les han sido denegadas sus peticiones, estando un $35,3 \%$ pendientes de respuesta, mientras que hay un 40,9\% que aún no han solicitado ninguna ayuda. Estos datos indican que, a nivel regional, las ayudas han tenido una gran acogida entre los emprendedores, el $60 \%$ las han solicitado.

Un análisis en función del tipo de empresa muestra que tan sólo existen diferencias significativas con la variable nivel de estudios.

Table 7. Desafios: financiación pública

\begin{tabular}{|l|c|c|c|c|c|c|c|c|c|}
\hline \multicolumn{1}{|c|}{ CLAVES } & \multicolumn{2}{c|}{ Tipo de iniciativa } & \multicolumn{2}{c|}{$\begin{array}{c}\text { Trabajadores } \\
\text { contratados }\end{array}$} & \multicolumn{3}{c|}{ Género } \\
& Total & $\begin{array}{c}\text { Nuevas } \\
\text { inic. }\end{array}$ & $\begin{array}{c}\text { Inic. } \\
\text { cons. }\end{array}$ & No & SI & Hombre & Mujer & No univ. & Univ. \\
\hline Estoy recibiendo ayudas públicas & $17,4 \%$ & $19,3 \%$ & $16,0 \%$ & $19,7 \%$ & $14,8 \%$ & $17,1 \%$ & $18,1 \%$ & $25,8 \% *$ & $14,5 \% *$ \\
\hline Estoy pendiente de respuesta & $35,3 \%$ & $37,6 \%$ & $33,6 \%$ & $30,7 \%$ & $40,7 \%$ & $40,7 \%$ & $35,8 \%$ & $37,1 \% *$ & $34,9 \% *$ \\
\hline Me han denegado & $6,4 \%$ & $2,8 \%$ & $9,6 \%$ & $7,9 \%$ & $4,6 \%$ & $4,6 \%$ & $9,6 \%$ & $3,2 \% *$ & $7,6 \% *$ \\
\hline No he solicitado & $40,9 \%$ & $40,4 \%$ & $40,8 \%$ & $40,7 \%$ & $39,8 \%$ & $42,8 \%$ & $37,3 \%$ & $33,9 \% *$ & $43,0 \% *$ \\
\hline
\end{tabular}

Fuente: Elaboración propia

p-valor: (*): p<0.1; (**): p<0.05; (***): $\mathrm{p}<0.01$

Se ha preguntado a los emprendedores acerca de si la situación actual puede propiciar que aparezcan nuevas oportunidades de mercado. Sobre la base de la Table 8, cabe destacar que hay un 62,3\% de emprendedores que consideran, con diferentes matices, que la situación actual puede propiciar que aparezcan nuevas oportunidades de mercado; de ellos sólo un 14\% opinan que aparecerán oportunidades con carácter general, un $22,5 \%$ asegura que las oportunidades serán para empresas con recursos suficientes y orientadas a actividades directamente relacionadas con la resolución de la crisis de la COVID-19 y, por último, un 25,8\% afirma que existirán para empresas con suficientes recursos y diversificadas.

Un 37,7\% son más pesimistas en cuanto a que surjan nuevas oportunidades pues aseguran que no surgirán oportunidades o sólo excepcionalmente.

Por último, respecto a las variables tipo de iniciativa, existencia de trabajadores, género y nivel de estudios del gerente, sólo existen diferencias estadísticas para esta última variable.

Table 8. Desafios: aparición de nuevas oportunidades

\begin{tabular}{|c|c|c|c|c|c|c|c|c|c|}
\hline \multirow[b]{2}{*}{ CLAVES } & \multirow[b]{2}{*}{ Total } & \multicolumn{2}{|c|}{ Tipo de iniciativa } & \multicolumn{2}{|c|}{$\begin{array}{l}\text { Trabajadores } \\
\text { contratados }\end{array}$} & \multicolumn{2}{|c|}{ Género } & \multicolumn{2}{|c|}{ Nivel estudios } \\
\hline & & $\begin{array}{l}\text { Nuevas } \\
\text { inic. }\end{array}$ & $\begin{array}{l}\text { Inic. } \\
\text { cons. }\end{array}$ & NO & SI & Hombre & Mujer & No univ. & Univ. \\
\hline Sí, para cualquier empresa & $14,0 \%$ & $16,5 \%$ & $11,9 \%$ & $13,4 \%$ & $14,8 \%$ & $15,1 \%$ & $11,9 \%$ & $6,3 \% * *$ & $16,9 \% * *$ \\
\hline $\begin{array}{l}\text { Sí, para empresas con recursos } \\
\text { suficientes y orientadas a actividades } \\
\text { relacionadas con la resolución de la } \\
\text { crisis }\end{array}$ & $22,5 \%$ & $24,8 \%$ & $19,8 \%$ & $22,8 \%$ & $22,2 \%$ & $23,7 \%$ & $22,2 \%$ & $28,6 \%$ ** & $19,8 \% * *$ \\
\hline $\begin{array}{l}\text { Sí, para empresas con recursos } \\
\text { suficientes y diversificadas }\end{array}$ & $25,8 \%$ & $21,1 \%$ & $30,2 \%$ & $25,2 \%$ & $26,9 \%$ & $23,0 \%$ & $31,0 \%$ & $17,5 \%$ ** & $29,1 \% * *$ \\
\hline $\begin{array}{l}\text { No o excepcionalmente, pero no con } \\
\text { carácter general }\end{array}$ & $37,7 \%$ & $37,6 \%$ & $38,1 \%$ & $38,6 \%$ & $36,1 \%$ & $38,2 \%$ & $36,9 \%$ & $47,6 \%{ }^{* *}$ & $34,3 \% * *$ \\
\hline
\end{tabular}

Fuente: Elaboración propia

p-valor: $(*): p<0.1 ;(* *): p<0.05 ;(* * *): p<0.01$

El análisis de los desafíos de la crisis COVID-19 termina con una descripción de la incidencia en el empleo y en las condiciones de trabajo en las empresas. La medida de urgencia más adoptada en materia laboral, adoptada por 1 de cada 2 empresas, es el teletrabajo (51,7\%). Entre las empresas que han podido mantener su actividad ha habido una baja incidencia de reducción de número de empleados $(14,4 \%)$, de horas de trabajo (14,4\%) y de recortes salariales $(8,1 \%)$. 
Table 9. Desafios: capital humano

\begin{tabular}{|c|c|c|c|c|c|c|c|c|c|}
\hline \multirow[b]{2}{*}{ CLAVES } & \multirow[b]{2}{*}{ Total } & \multicolumn{2}{|c|}{ Tipo de iniciativa } & \multicolumn{2}{|c|}{$\begin{array}{l}\text { Trabajadores } \\
\text { contratados }\end{array}$} & \multicolumn{2}{|c|}{ Género } & \multicolumn{2}{|c|}{ Nivel estudios } \\
\hline & & $\begin{array}{l}\text { Nuevas } \\
\text { inic. }\end{array}$ & $\begin{array}{l}\text { Inic. } \\
\text { cons. }\end{array}$ & NO & SI & Hombre & Mujer & No univ. & Univ. \\
\hline Reducción del número de empleados & $14,4 \%$ & $7,3 \% * * *$ & $20,6 \% * * *$ & $1,6 \% * * *$ & $29,6 \% * * *$ & $17,8 \% * * *$ & $8,3 \% * * *$ & $12,7 \%$ & $15,1 \%$ \\
\hline $\begin{array}{l}\text { Reducción de las horas de trabajo } \\
\text { personal }\end{array}$ & $14,4 \%$ & $9,2 \% * *$ & $19,0 \% * *$ & $7,1 \% * * *$ & $23,1 \% * *$ & $15,8 \%$ & $11,9 \%$ & $11,1 \%$ & $15,7 \%$ \\
\hline Recortes salariales & $8,1 \%$ & $8,3 \%$ & $7,9 \%$ & $7,9 \%$ & $8,3 \%$ & $10,5 \% *$ & $3,6 \% *$ & $9,5 \%$ & $7,6 \%$ \\
\hline Teletrabajo & $51,7 \%$ & $45,9 \% *$ & $57,1 \% *$ & $44,1 \% * * *$ & $61,1 \% * * *$ & $58,6 \% * * *$ & $39,3 \% * * *$ & $20,6 \% * * *$ & $63,4 \% * * *$ \\
\hline
\end{tabular}

Fuente: Elaboración propia

p-valor: $(*): \mathrm{p}<0.1 ;(* *): \mathrm{p}<0.05 ;(* * *): \mathrm{p}<0.01$

Si miramos el tipo de iniciativa y el perfil del gerente (Table 9), la reducción del número de empleados y horas se ha realizado en mayor medida en las empresas consolidadas. Los hombres, además de reducir las horas de sus trabajadores en mayor medida que las mujeres, han realizado más recortes salariales y fomentan más el teletrabajo. Por último, los gerentes con estudios universitarios han fomentado más el teletrabajo.

\section{Conclusiones}

Actualmente, como muchos países, España se encuentra saliendo de la situación de emergencia de salud pública que el Gobierno decretó debido a la pandemia por la COVID-19. Esto ha provocado una paralización de la economía, ocasionado el confinamiento obligatorio que se ha tenido que realizar a nivel mundial. Por ello, los gobiernos han tenido que desarrollar cantidad de medidas para limitar sus graves consecuencias entre la población como fueron rentas mínimas, ayudas a autónomos, ERTEs, entre otras.

Todo parece indicar que, pese al esfuerzo institucional, esta crisis sanitaria provocará una intensa crisis económica. Como en crisis anteriores, el papel del tejido empresarial y, principalmente, de los emprendedores es destacado por los gobiernos considerándolos el pilar esencial en el que se tiene que sostener la economía del país para poder salir de la crisis.

Sin embargo, el hecho de que esta crisis esté provocada por una pandemia que afecta a nivel mundial y que esta, a su vez, haya provocado el confinamiento de la población de gran cantidad de países, hace ver que la situación ocasionada por la actual crisis es diferente a las vividas hasta el momento. Este hecho concreto hace que los investigadores se centren en analizar desafíos a los que se tienen que enfrentar los emprendedores ante esta crisis de la COVID-19.

En concreto, entre los desafíos a los que se tienen que enfrentar los emprendedores se habla de la digitalización, ya que, al estar las personas confinadas, muchos servicios y tratos entre empresas y clientes se debían hacer a través de diferentes plataformas de videoconferencias, por ejemplo. El segundo de los desafíos tiene que ver con la financiación de los emprendedores, ya que resulta obvio que si estos no venden no pueden tener ingresos, sin embargo, es destacable el esfuerzo realizado por los gobiernos para poder ayudar a los emprendedores a superar este desafío. El tercer desafío sería el de la percepción de oportunidades, no obstante, sobre este se debe poner de manifiesto que la literatura identifica los periodos de crisis como el momento idóneo para que los individuos puedan encontrar oportunidades innovadoras. Finalmente, se destaca el desafío del capital humano, ya que muchas empresas han visto que existía escasez de trabajadores (como es el caso del sector sanitario), mientras que su opuesto se encuentran en la mayoría de empresas que, al tener que cerrar sus establecimientos, se han visto abocadas a solicitar un ERTE por no poder mantener los costes salariales durante el confinamiento.

Todo esto hace pensar que resulta necesario diseñar unas nuevas líneas de actuación apropiadas al actual, complejo e incierto contexto en el que nos encontramos. Para ello, se debe hacer uso de todos los recursos disponibles y, con ellos, se puede ayudar a los individuos a identificar y explotar oportunidades de negocio.

Los resultados del análisis realizado muestran, en primer lugar, una fuerte ralentización de la actividad empresarial. Sin duda, influida directamente por los altos niveles de incertidumbre ante un escenario nuevo para todos. Los cambios sobrevenidos han sido la norma, sólo un 13,1\% ha seguido operando normalmente. La antigüedad de la empresa y el hecho de tener trabajadores contratados ha favorecido la normalidad en aquellas empresas que no han tenido que cerrar por orden administrativa. Esto sin duda se debe a que se trata de iniciativas más fuertes y consolidadas. La formación del gerente también ha sido clave para la continuidad de la empresa. La velocidad e intensidad de los cambios aumenta la importancia de las habilidades, conocimientos y experiencias del directivo en todo tipo de empresas. La ausencia de capacidad en el directivo es una de las principales causas de fracaso en las pequeñas y medianas empresas (Ahire \& Golphar, 1996).

Sin duda, el cambio más relevante en el ámbito laboral es el teletrabajo, 1 de cada 2 empleados ha trabajado de forma remota desde sus domicilios. Una opción que, antes del estado de alarma, solía constituir una 
alternativa puntual y minoritaria. Esta opción es más marcada en las empresas más asentadas en el mercado y en aquellas cuyos gerentes tienen formación universitaria. Esto puede deberse a que, a medida que la empresa va operando, puede acometer inversiones que favorezcan la digitalización. Del mismo modo, la formación universitaria acerca a los emprendedores a la tecnología necesaria para el teletrabajo.

Los cambios en la demanda de bienes y servicios han afectado por igual a todas las empresas. Concretamente, un tercio de ellas no ha tenido demanda en el período del estado de alarma analizado, por permanecer cerrada su actividad. Por el contrario, un 19,8\% ha mantenido constante su demanda o incluso la ha visto aumentada. Estos efectos tan negativos en la demanda de bienes y servicios se producen por dos cuestiones. En primer lugar, por el enorme vacío de expectativas, tanto a nivel social como empresarial, generado por la ausencia de un horizonte claro. En segundo lugar, este periodo de crisis ha ocasionado una pérdida de ingresos en las familias que ha llevado a la consiguiente disminución del consumo y a unos mayores riesgos de morosidad.

Asimismo, la incidencia de la crisis COVID-19 en el empleo y en las condiciones de trabajo en las empresas de la muestra indica que las situaciones expuestas anteriormente han tenido su reflejo en los empleados por cuenta ajena. Si bien el 46,3\% afirma no haber realizado expedientes de regulación de empleo o despidos, el resto sí han tenido que adoptar estas medidas laborales de urgencia. Medidas que han afectado menos a las iniciativas nuevas. Esto puede deberse a que, concretamente, estas empresas son las que menos trabajadores tienen contratados. De hecho, el 63,8\% no tenía ningún empleado.

Respecto a las dificultades de los emprendedores en este periodo resalta la preocupación de no poder superar un periodo de aislamiento tan largo (67,8\%). A esta le sigue el miedo a asumir riegos (57,2\%) y el temor a que se habiliten líneas de financiación públicas suficientes $(53,4 \%)$.

Es necesario poner en marcha medidas para que las iniciativas nuevas puedan llegar a consolidarse y mantenerse. Una situación prolongada de crisis podría llevar, no sólo a agravar estos miedos, lo que llevaría implícito la desaparición de muchas iniciativas emprendedoras. El miedo es una importante barrera a la explotación de las oportunidades percibidas. Si el emprendedor tiene miedo y percibe que la opción de crear una empresa es demasiado arriesgada, no se embarcará en esa aventura. Por ello, las administraciones deben seguir trabajando en facilitar las líneas de financiación que se constituyan en los pilares necesarios para, además de lograr mantener el empleo, fomenten la innovación y la inversión necesaria para paliar los efectos económicos de la crisis sanitaria.

Los desafíos que se proponen para la reflexión se agrupan en cuatro grandes categorías: la importancia de la digitalización de las empresas, el acceso a la financiación pública, la aparición de oportunidades y el esencial papel de los recursos humanos para afrontar los retos competitivos.

La crisis ha convertido a la digitalización en una prioridad para hacer frente a los retos y oportunidades derivados de las consecuencias de la crisis de la COVID-19. Los empleados se han familiarizado con el uso de plataformas para compartir archivos, tecnología que permite pasar un documento de un lugar a otro sin necesidad de estar en contacto físico con el receptor, o con las redes internas virtuales de trabajo que permiten mandar información de forma encriptada, garantizar la confidencialidad de archivos y datos y facilitar el acceso a contenidos online y páginas web. Pero sin duda, la herramienta que más protagonismo ha adquirido ha sido la videoconferencia, la utilizan uno de cada dos emprendedores. Esta herramienta ha permitido la comunicación sincrónica y bidireccional de audio y vídeo, promoviendo reuniones con grupos de personas situadas en lugares alejados entre sí.

Es necesario también un apoyo en este sentido. Las administraciones públicas deben pensar en articular ayudas para la adquisición de equipamiento que permita incrementar la digitalización. Asimismo, se debe fomentar la adquisición de licencias a través de clústeres de emprendedores, así como la formación necesaria de los mismos para el mejor uso de las nuevas herramientas de gestión que se irán implantando -tanto en comunicación como en gestión del trabajo y en las relaciones con los clientes-.

El desarrollo de infraestructuras y la formación en nuevas tecnologías es una necesidad urgente en todas las empresas, pero en especial en las empresas más jóvenes y más pequeñas, las dirigidas por mujeres y personas con bajos niveles de formación. Pues en ellas, según los resultados obtenidos, la implantación de las nuevas tecnologías es menor.

El siguiente desafío tiene que ver con los recursos financieros disponibles. La supervivencia de muchas empresas va a depender de la financiación. Se va a necesitar dinero para sobrevivir, para hacer frente a ajustes de plantilla y para acometer distintas inversiones. Son muchas las fórmulas financieras diseñadas por el colectivo de sociedades de garantía recíproca (garantías y avales), instituciones financieras tradicionales (facilidades para las líneas de crédito) y de las administraciones públicas (programas de subsidios). De hecho, más del $60 \%$ de los emprendedores las han solicitado y el 17,4\% ya las están recibiendo. Esto va a favorecer la inyección de liquidez en el sistema y a facilitar los planes de inversión y reestructuración. 
Aunque no se aprecian diferencias significativas en función del tipo de empresa, sin duda las ayudas y medidas deberían ajustarse a las circunstancias de los diferentes tamaños empresariales y son especialmente relevantes en las actividades que han estado más tiempo paralizadas.

Por otro lado, es fundamental seguir fomentando la cultura emprendedora para poder explotar las nuevas oportunidades. Los emprendedores suelen contar con una fuerte intuición a la hora de identificar nichos de negocio rentables y suelen apostar por sus ideas, al margen de que la supuesta viabilidad no sea observada por el resto de agentes. En este sentido, los resultados muestran que los emprendedores esperan tener oportunidades derivadas de esta situación sobrevenida. Concretamente así lo afirman el 62,3\% de los encuestados. Esto lo dicen, por una parte, unido a que puedan contar con suficientes recursos (financieros, humanos, infraestructura, etc.) y a la diversificación; y, por otro lado, a que se cuenten con nuevas oportunidades directamente relacionadas con la resolución de la crisis de la COVID-19 -es decir, oportunidades nuevas que se podrán aprovechar desde una posición de fortaleza en su dotación de recursos-, obviando a los más pesimistas, ese $37,7 \%$ que no ven posibilidad de nuevas oportunidades en el futuro.

Pensando en la recuperación, es clave que la intención emprendedora no disminuya. Esta capacidad de identificar oportunidades que el estudio ha recogido es, sin duda, la semilla de nuestra prosperidad futura. Por tanto, y aún siendo conscientes del momento tan complicado que estamos viviendo, los resultados también nos invitan a un sereno optimismo respecto al futuro y a una fe en nuestra capacidad de reactivar la actividad. En este sentido, habrá que apoyar al emprendedor desde la administración, pues no hacerlo podría provocar una recuperación más lenta cuando disminuyan los problemas sanitarios.

El último desafío analizado es el relacionado con el capital humano. La suspensión temporal de contratos, las reducciones temporales de jornada y los despidos y extinciones de contratos se han generalizado tal y como se ha comentado. Ha estas medidas se han unido reducciones de jornadas y recortes salariales. Prácticamente 1 de cada 2 emprendedores ha perdido capital humano, de forma definitiva (mediante despidos) o temporalmente. Todas estas medidas, en primer lugar, agudizan la crisis al suponer una reducción de ingresos para los empleados y una caída del ahorro individual que, sin duda, tendrá fuertes implicaciones económicas. En segundo lugar, un aspecto esencial es tanto la caída de la moral y de la motivación de los que se quedan y una fuerte pérdida de talento organizativo.

Es el momento de recordar el rol jugado por los recursos humanos como factor competitivo decisivo. Pese a la situación que están atravesando los emprendedores, no deben olvidar establecer las estrategias necesarias para asegurar una mano de obra motivada, formada y capaz de producir con calidad. Olvidar esto no sólo reducirá la productividad y la rentabilidad, sino que puede crear un clima negativo que desemboque incluso en el cierre de los negocios. Así mismo, deben saber que la reactivación va a estar muy condicionada por la posibilidad de poder disponer con rapidez del capital humano necesario para seguir impulsando el negocio.

En resumen, todo parece indicar que la crisis sanitaria de la COVID-19 está provocando una grave crisis económica, debido a la paralización de la economía ocurrida por el confinamiento al que ha estado sometida la población de la mayoría de países del mundo. Al analizar la literatura existente, si algo esta pone de manifiesto es el hecho de que, como en otras crisis anteriores, los emprendedores tienen un papel fundamental para afrontar esta especial situación y provocar la recuperación económica. De hecho, es cierto que en la literatura se indica que es posible percibir oportunidades innovadoras en mayor medida en periodos de crisis, sin embargo, no se debe pensar que todo lo que conlleva una crisis económica hace que emprender sea más sencillo. Por ello, se afirma que los emprendedores necesitan apoyo institucional para poder crear o continuar con su organización tras esta crisis provocada por la COVID-19, al tener que enfrentarse a importantes desafíos como son las necesidades de digitalización, falta de financiación, disminución del capital humano o percibir oportunidades como posibles negocios viables.

\section{References}

Ahire, S. L., \& Golphar, D. Y. (1996). Quality management in large vs small firms. Journal of Small Business Management, 34(3), 1-13

Baron, R. A. (2004). The cognitive perspective: a valuable tool for answering entrepreneurship's basic "why" questions. Journal of Business Venturing, 19(2), 221-239. https://doi.org/10.1016/S0883-9026(03)00008-9

Brindle, M. E., \& Gawande, A. (2020). Managing COVID-19 in Surgical Systems. Annals of Surgery, 272(1), e1-e2. https://doi.org/10.1097/SLA.0000000000003923

CEOE (2020). Estrategia España para la recuperación de la crisis del COVID-19. CEOE. Retrieved from https://contenidos.ceoe.es/CEOE/var/pool/pdf/publications_docs-file-794-estrategia-espana-para-larecuperacion-de-la-crisis-del-covid-19.pdf

Cantillon, R. (1978). Ensayo sobre la naturaleza del comercio en general. México: Fondo de Cultura Económica 
Constant, A., \& Zimmermann, K. F. (2006). The Making of Entrepreneurs in Germany: Are Native Men and Immigrants Alike?. Small Business Economics, 26(3), 279-300.

https://doi.org/10.1007/s11187-005-3004-6

Goodell, J. W. (2020). COVID-19 and finance: Agendas for future research. Finance Research Letters, 35. https://doi.org/10.1016/j.frl.2020.101512

Gutiérrez Solana, F., Fernández-Laviada, A., Peña, I., Guerrero, M., González-Pernía, J. L., Montero, J., ... Sánchez de León, J. D. N. (2018). Global Entrepreneurship Monitor. Informe GEM España 2017-2018. Santander, España: Editorial de la Universidad de Cantabria. https://doi.org/10.22429/Euc2018.064

Haeffele, S., Hobson, A., \& Storr, V. H. (2020). Coming Back from Covid-19: Lessons in Entrepreneurship from Disaster Recovery Research. SSRN Electronic Journal. https://doi.org/10.2139/ssrn.3592966

Jiménez Zarco, A. I. (2012). Emprender en tipos de crisis: emprendimiento innovador como base de generación de valor. Paper presented at VIII Congreso Virtual Emprendimiento, PYMES y Gestión del Conocimiento, Bogotá, Colombia

Leibenstein, H. (1968). Entrepreneurship and development. The American Economic Review, 58(2), 72-83. Retrieved from https://www.jstor.org/stable/1831799

Liñán, F. (2004). Educación empresarial y modelo de intenciones. Formación para un empresariado de calidad. (Doctoral dissertation).. Universidad de Sevilla. Retrieved from http://hdl.handle.net/11441/15036

Ministerio de Trabajo (2009). Cuadernos del Mercado de Trabajo, Revista del Ministerio de Empleo, Edición semestral, $n^{\underline{o}} 2$.

Morcillo, F. (2005). Economía: teoría y política. Barcelona, Spain: McGraw-Hill. Retrieved from https://www.researchgate.net/profile/Francisco_Mochon/publication/27298655_Economa_Teora_y_Poltica /links/544763600cf2d62c305064a1.pdf

Muñoz Palacios, G. (2017). Emprender: alternativa profesional en la crisis del siglo XXI. Universidad de Valladolid. Retrieved from http://uvadoc.uva.es/handle/10324/27439

Nicolás Martínez, C., Rubio Bañón, A., \& Fernández Laviada, A. (2019). Social Entrepreneur: Same or Different from the Rest?. VOLUNTAS: International Journal of Voluntary and Nonprofit Organizations, 30(3), 443-459. https://doi.org/10.1007/s11266-018-00053-9

Nicolás, C., \& Rubio, A. (2015). Gestión de recursos humanos en la empresa social. Universia Business Review, 47, 82 - 105. Retrieved from https://journals.ucjc.edu/ubr/article/view/1508

Ortega, E. (2019). Este mapa muestra cuánto tardó cada país europeo en recuperar el PIB per cápita anterior a la crisis de 2008. Busines Inside España. Retrieved from https://www.businessinsider.es/maparecuperacion-crisis-2008-paises-europeos-517617

Parra Miranda, M. S. (2014). Emprender en tiempos de crisis. Observatorio Económico, 80, 2-3. https://doi.org/10.11565/oe.vi80.130

Peñaherrera León, M., \& Cobos Alvarado, F. (2012). La creatividad y el emprendimiento en tiempos de crisis. Revista Iberoamericana sobre Calidad, Eficacia y Cambio en Educación, 10(2), 238-247. Retrieved from https://revistas.uam.es/reice/article/view/3066

Schumpeter, J. (1911). Theory of Economic Development. New York, USA: Oxford University Press

Zander, I. (2004). El espíritu emprendedor en el ámbito geográfico. Fundamentos conceptuales e implicaciones para la formación de nuevos clusters. Cuadernos de economía y dirección de la empresa, $20,9-34$

Zhang, D., Hu, M., \& Ji, Q. (2020). Financial markets under the global pandemic of COVID-19. Finance Research Letters, 101528. https://doi.org/10.1016/j.frl.2020.101528 